\title{
Einführung von Telekooperation in der Landeshauptstadt Stuttgart
}

Majer, Andreas ; Schwabe, Gerhard

Posted at the Zurich Open Repository and Archive, University of Zurich ZORA URL: https://doi.org/10.5167/uzh-67793

Conference or Workshop Item

Originally published at:

Majer, Andreas; Schwabe, Gerhard (1998). Einführung von Telekooperation in der Landeshauptstadt Stuttgart. In: Hermann, T.; Just-Hahn, K.:Groupware und organisatorische Innovation, Tagungsband der Deutschen Computer Supported Cooperative Work - DCSCW 98, Wiesbaden, 1 January 1998, Teubner. 


\title{
Einführung von Telekooperation in der Landeshauptstadt Stuttgart
}

\author{
Andreas Majer, Landeshauptstadt Stuttgart, \\ Gerhard Schwabe, Universität Hohenheim
}

\section{$1 \quad$ Einleitung}

Die Landeshauptstadt Stuttgart befindet sich im Umbruch: Die steigenden Haushaltsausgaben und die sinkenden Haushaltseinnahmen seit Anfang der 90er Jahre sind nicht mehr nur durch reine Sparmaßnahmen aufzufangen. Vielmehr müssen die Arbeitsprozesse in der Stadtverwaltung reorganisiert und die Leistungsfähigkeit der Mitarbeiter und Mitarbeiterinnen auf allen Ebenen erhöht werden. Hierzu wird auch auf den Einsatz von Telekooperation gesetzt (vgl. z.B. [Landeshauptstadt Stuttgart 1998, Murawski 1998]). Telekooperative Anwendungen werden in der Landeshauptstadt Stuttgart in erster Linie mit Lotus Notes/Domino realisiert, womit die Zusammenarbeit zwischen räumlich verteilten Personen, Abteilungen, Ämtern und Referaten unterstützt wird.

In diesem Beitrag werden am Beispiel von vier Lotus Notes/Domino-Anwendungen Einführungserfahrungen und Einführungskonzepte vorgestellt: Zu Beginn wird ein Gesamtkonzept für eine Einführungsstrategie vorgestellt. An diesem Gesamtkonzept wird deutlich, daß für verschiedene Führungsebenen unterschiedliche Einführungsstrategien sinnvoll sind, aber der Wettbewerb auf allen Ebenen (wenn auch nicht auf allen Ebenen gleich) als Einführungsstrategie eingesetzt werden kann. Anschließend werden in eigenen Kapiteln die Einführung der Cuparla-Lösung für den Gemeinderat, die Planung der Einführung eines TelemanagerSystems für die Verwaltungsspitze, die erfolgte Einführung eines kommunalen Sitzungsdienstes auch für die mittlere Führungsebene und das gerade aktuell in der Einführung befindliche System für einen mobilen Benutzerservice vorgestellt.

\section{Gesamtkonzept für die Einführung von Telekooperation in der LHS Stuttgart}

Die Einführung von Telekooperation in der Landeshauptstadt Stuttgart ist die Aufgabe eines DV-Organisators; in der privaten Wirtschaft würde man seine Aufgaben dem Informationsmanagement zuordnen (vgl. [Krcmar 1997, Schwabe 1998b]. Seine Arbeit hat primär 
einen organisatorischen Fokus: Die Organisation soll an neue, externe Anforderungen angepaßt werden; die Technologie ist der Hebel, mit dem diese organisatorischen Maßnahmen umgesetzt werden können. Deshalb ist es nicht verwunderlich, daß die Einführung von Telekooperation dann an organisatorischen Hindernissen scheitern kann. Der DVOrganisator benötigt eine geeignete Einführungsstrategie, um diese Hindernisse zu umschiffen. Diese Einführungsstrategie hat zwei Dimensionen: Eine öffentliche, rationalökonomisch geprägte Dimension und eine im mehr Untergrund wirkende, mikropolitische Dimension (zur Mikropolitik bei der Einführung von Informationssystemen vgl. [Ortmann et al. 1990]): Rationalökonomisch wird mit Zeitersparnissen, Qualitätsverbesserung und Kostenersparnis argumentiert; mikropolitisch stehen beispielsweise die persönliche Profilierung, Macht, Risikovermeidung ("nur nichts falsch machen") und die möglichst gute Vereinbarkeit von Beruf und Privatleben im Vordergrund ${ }^{1}$.

Eine Einführungsstrategie muß immer an beiden Größen zugleich ansetzen: Sie muß sowohl rationalökonomisch begründet als auch den mikropolitischen Interessen der Akteure entgegenkommen. Sowohl die rationalökonomische Argumentation als auch die mikropolitischen Interessen der beteiligten Akteure hängen von der Rolle und der hierarchischen Position der Akteure ab. Abbildung 1 stellt das Zusammenwirken von rationalökonomischer Dimension und von mikropolitischer Dimension exemplarisch dar. Es sei aber vorausgeschickt, daß diese Grafik bewußt verkürzt und überzeichnet ist, um das Zusammenwirken der beiden Dimensionen und den Ansatzpunkt für eine Einführungsstrategie darzustellen und in dieser Deutlichkeit die Situation in Stuttgart nicht beschreibt.

\footnotetext{
${ }^{1} \mathrm{Zu}$ den theoretischen Implikationen der Unterscheidungn zwischen der rationalökonomischen und der mikropolitischen Perspektive vgl. [Schwarzer et al. 1995].
} 


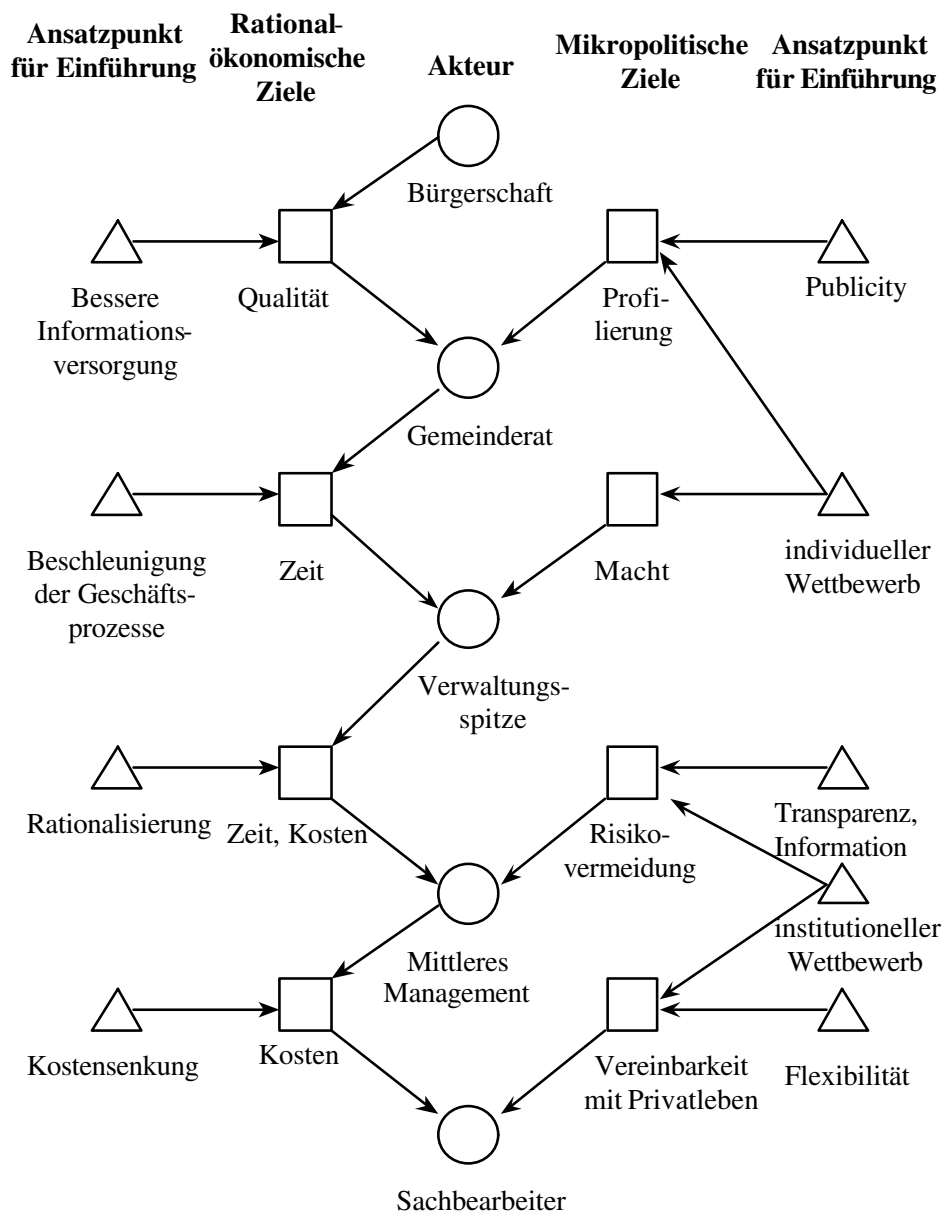

Abbildung 1: Ansatzpunkte für eine Einführungsstrategie

Die Bürgerschaft als Gesamtheit fordert vom Gemeinderat Qualität bei seinen Entscheidungen. Telekooperationstechnologie kann die Erreichung dieses Qualitätsziels rationalökonomisch dadurch fördern, daß es die Informationsversorgung verbessert. Das mikropolitische Ziel jedes einzelnen Stadtrats ist seine Profilierung, denn in Baden-Württemberg werden die Stadträte in einem Personenwahlrecht gewählt. Ein mikropolitischer Ansatzpunkt liegt demnach darin, daß man den einzelnen Stadträten mit der Einführung Publicity verschafft und somit zu ihrer Profilierung beiträgt. Zu ihrer Profilierung kann man auch beitragen, indem man ihnen einen individuellen Wettbewerbsvorteil gegenüber den anderen Gemeinderatsmitgliedern gewährt.

Der Gemeinderat gibt der Verwaltungsspitze mit den Aufgaben häufig ein Zeitziel vor, bis zu denen die Aufgabe erledigt sein soll. Dieses Zeitziel - ein rationalökonomisches Ziel kann durch die Beschleunigung der Geschäftsprozesse besser erreicht werden. Deshalb ist 
die Verwaltungsspitze dann für die Einführung von Telekooperation besonders empfänglich, wenn sie sich eine Beschleunigung der Geschäftsprozesse verspricht. Als individuelle Ziele auf der mikropolitischen Ebene strebt die Verwaltungsspitze Machterhalt und Machtausweitung an. Deshalb sind diese Führungskräfte für Telekooperation besonders empfänglich, wenn sie über einen individuellen Wettbewerbsvorteil ihre Machtposition verbessern können.

Die Verwaltungsspitze gibt dem Mittleren Management typischerweise Zeit- und Kostenziele vor. Rationalökonomisch sinnvoll ist Telekooperationstechnologie auf dieser Ebene dann, wenn sie zur Rationalisierung beiträgt. Diese Führungsebene ist häufig sehr risikoscheu. Ihrem Drang nach Risikovermeidung kann Telekooperationstechnologie dadurch entgegenkommen, daß sie Transparenz über die gerade laufenden Aktivitäten in dem Bereich der Führungskraft und an den ihn betreffenden anderen Bereichen herstellt. Dann lassen sich Risiken rechtzeitig erkennen und auch vermeiden. Wirkungsvoll ist auf dieser Ebene auch der institutionelle Wettbewerb, indem z.B. angedroht wird, Aktivitäten über Aufträge an freie Unternehmen auszulagern oder ganze Abteilungen über Outsourcing dem Marktdruck auszusetzen.

Das mittlere Management gibt insbesondere die Kostenziele an die Sachbearbeiter weiter. Deshalb ist Telekooperation auf dieser Ebene rationalökonomisch sinnvoll, wenn sie zu Kostensenkungen führt. Das mikropolitische Ziel des Sachbearbeiters (der keine Führungsposition anstrebt) ist eine möglichst gute Vereinbarkeit von Beruf und Privatleben. Durch einen institutionellen Wettbewerb kann er dieses Ziel gefährdet sehen und ist dann bereit, zur Abwehr der "Gefahr" Telekooperation einzusetzen. Telekooperation kann aber auch direkt die Vereinbarkeit von Beruf und Privatleben verbessern, indem sie dem Sachberarbeiter räumliche und zeitliche Flexibilität einräumt.

\section{Einführung von Telekooperation für den Gemeinderat}

Gemeinderäte sind zeitlich sehr stark belastet. Neben dem Hauptberuf sind sie ehrenamtlich z.T. mehr als 40 Stunden pro Woche für die Kommune tätig. Da sie in der Verwaltung im Regelfall kein Büro haben, müssen sie sich abends und am Wochenende zu Hause auf Ihre Sitzungen und Entscheidungen vorbereiten. Zu dieser Zeit sind in der in der kommunalen Verwaltung und den Fraktionsgeschäftsstellen keine Mitarbeiter verfügbar. Das Projekt Computerunterstiutzung der Parlamentsarbeit (Cuparla) ${ }^{2}$ versetzt sie in die Lage, mit Hilfe eines Notebooks Gemeinderatsarbeit zu Hause und an jedem anderen Ort durchzuführen

\footnotetext{
${ }^{2}$ Das Projekt Cuparla wurde von Universität Hohenheim (Projektkoordinator), der Datenzentrale Baden-Württemberg und der ITM GmbH im Auftrag der Deutschen Telekom Berkom GmbH von 1995-1997 durchgeführt. Seit Anfang 1998 arbeitet die LHS Stuttgart mit Unterstützung der Projektpartner in Eigenregie daran weiter.
} 
und dabei auf Informationsbestände der Verwaltung, der Fraktion und von anderen Gemeinderatsmitgliedern zuzugreifen (für eine umfassende wissenschaftliche Behandlung von Cuparla vgl. [Schwabe 1998]).

In Stuttgart wurden im Laufe des Jahres 199756 von 60 Gemeinderäten mit Notebooks ausgestattet. Auf den Notebooks befindet sich eine Telekooperationsumgebung auf der Basis von Lotus Notes und GroupSystems (vgl. Schwabe et al. 1997, Schwabe\&Vöhringer 1998]. Dadurch können die Gemeinderäte mobil auf die Informationsbestände der Verwaltung zugreifen und innerhalb der Fraktion miteinander per Email kommunizieren und kooperieren.

Dadurch soll für den Anwender

- die Gemeinderatsarbeit effizienter und flexibler gestaltet,

- der Informationszugang der Gemeinderäte verbessert und

- Kommunikations- und Kooperationsbarrieren innerhalb des Parlaments und zwischen Parlament und Verwaltung abgebaut werden.

Das Projekt Cuparla bringt der Stadt Stuttgart vermutlich langfristig den größten Nutzen durch die Optimierung der Geschäftsprozesse in der Verwaltung sowie an der Schnittstelle zum Gemeinderat. Bei aller öffentlichen Rhetorik scheint dieses eher ökonomische Kriterium für die betroffenen Entscheidungsträger (Stadträte, Bürgermeister ...) kein besonderer Anreiz zu sein, Telekooperationstechnologie persönlich zu nutzen. Interessant wird es dann für den einzelnen Entscheidungsträger, wenn er einen erfahrbaren, individuellen Nutzen davon hat. Einer der stärksten Anreize für einen Entscheidungsträger ist der Wettbewerbsvorteil gegenüber einem Mitwettbewerber oder die Beseitigung eines Wettbewerbsnachteils.

Diese These soll im folgenden durch Beobachtungen untermauert werden, die wiederum in Thesen verdichtet werden. Der Wettbewerb spielt auf kommunaler Ebene eine große Rolle: Der einzelne Stadtrat steht im Wettbewerb um Listenplätze, Stimmen und Ämter. Die Parteien stehen in einem Wettbewerb um Stimmen und die Macht auf kommunaler Ebene. Charakteristisch für die Kommunalpolitik ist ein Wettbewerb zwischen Gemeinderat und Verwaltung um jeweils Freiräume für die bessere Gestaltung der Stadtpolitik zu erhalten sowie der Wettbewerb zwischen Kommunen (Standortpolitik). Die Betonung des Wettbewerbs auf allen vier Ebenen stellte in Cuparla einen wesentlichen Faktor für die erfolgreiche Einführung der Telekooperationstechnologie dar. Die Wirkung des Wettbewerbs sei in einer Gesamtbeobachtung zusammengefaßt, die durch vier Teilbeobachtungen gestützt wird (vgl. [Schwabe\&Krcmar 1998]:

Gesamtbeobachtung: Der Wettbewerb stellt für Entscheidungsträger einen wesentlichen Anreiz für die Nutzung von Telekooperationstechnologie dar.

Teilbeobachtung W1 zum Wettbewerb auf individueller Ebene: Größere Unabhängigkeit bei der Informationsbeschaffung beseitigt einen Wettbewerbsnachteil für einen Entscheidungsträger; besserer Informationszugang und bessere Vernetzungsfähigkeit bei der Umsetzung seiner Anliegen bringen ihm einen Wettbewerbsvorteil. 
Teilbeobachtung W2 zum Wettbewerb auf Fraktionsebene: Eine Verbesserung der Kampagnefähigkeit führt zu einem Wettbewerbsvorteil für eine Fraktion.

Teilbeobachtung W3 zum Wettbewerb auf Organisationsebene: Eine Verbesserung der Informationsversorgung führt zu einer Vergrößerung der Handlungsspielräume für den Entscheidungsträger (Gemeinderat).

Teilbeobachtung W4 zum Wettbewerb auf interkommunaler Ebene: Effizientere Entscheidungsprozesse in einer Kommune sind ein nach außen sichtbarer Standortvorteil.

\section{$4 \quad$ Einführung eines telekooperativ nutzbaren Führungsinformationssystems: Telemanager}

Die Informationsversorgung des Gemeinderats der Landeshauptstadt Stuttgart wurde durch Cuparla quantitativ und qualitativ erheblich verbessert. Hinzu kommt eine Beschleunigung in der Bereitstellung von Informationen. Dieser „Vorsprung“ für das oberste Organ der Landeshauptstadt Stuttgart ist zwangsläufig für die Verwaltungsführung nicht ganz unproblematisch. Anträge und Anfragen des Cemeinderats können durch die Nutzung der Möglichkeiten von Cuparla wesentlich konkreter als bisher gestellt werden. Zudem wurden durch den Einsatz von Telekooperationstechnologie gemeinsame Ad-hoc-Initiativen einzelner Stadträte oder von Fraktionen erheblich vereinfacht. Die Verwaltungsführung, die die Antwortschreiben erstellen muß, benötigt dagegen zahlreiche Mitarbeiter, die sie bei der Recherche nach den notwendigen Informationen unterstützt. Der Verwaltung verliert damit zwangsläufig ihren „Exklusivzugriff“ auf den eigenen Informationsbestand. Dies ist auch in der politischen Auseinandersetzung während der Sitzungen zu beobachten: Jeder einzelne Stadtrat kann bequem über die Volltextsuche in Vorlagen und Protokollen recherchieren. Die Verwaltungsführung muß dagegen häufig erst ihren „Apparat“ in Bewegung setzen, um zumeist nach der Sitzung Argumente bereitzustellen.

Cuparla hat somit ein über viele Jahre gewachsenes Gleichgewicht in einem konstruktiven Sinne verschoben und für eine - zur Zeit noch einseitige - Verbesserung der Informationsbereitstellung gesorgt.

Dieses Gleichgewicht kann durch den Einsatz von Telekooperationstechnologie für Bürgermeister und Amtsleitungen wieder hergestellt werden. Dies aber nicht aus einer Lust am „technologischen Abenteuer“. Vielmehr stehen die Ziele der Verwaltungsreform im Mittelpunkt. Bürgerorientierung, um nur eines dieser Ziele zu nennen, wird für Gemeinderat und Verwaltung zur gemeinsamen Meßlatte [Landeshauptstadt Stuttgart 1998]. Die Bürger werden eine bessere Effizienz von Gemeinderatssitzungen verlangen, wollen die Ergebnisse und Leistungen ihrer gewählten, ehrenamtlich tätigen Stadträte wie auch der Bürgermeister und der gesamten Verwaltung besser kontrollieren können. Stadträte wie Bürgermeister benötigen aber zunächst den direkten Zugriff auf alle entscheidungsrelevanten Informationen - und zwar an jedem Ort und möglichst zu jeder Zeit. Dies führte zu der Projektidee des „Telemanagers“, die Telekooperation und Führungsinformation für die Verwaltungsführung beinhalten soll: 


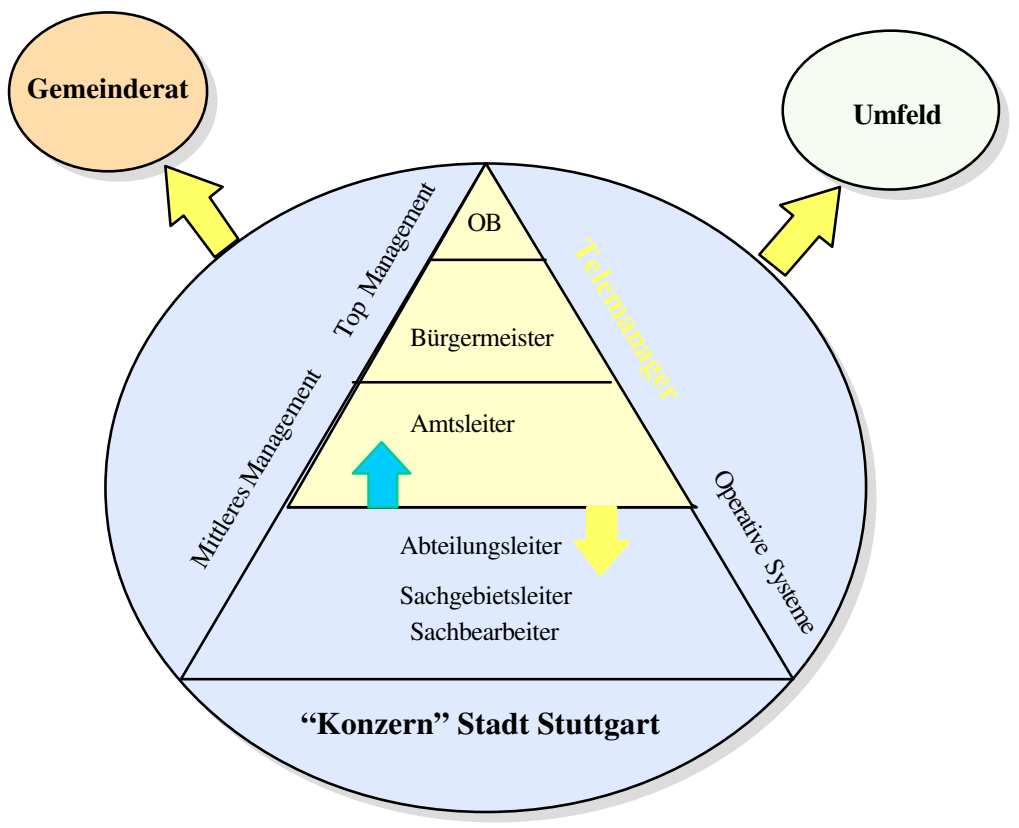

Abbildung 2: Einordnung des Telemanagers

Mit Telemanager sind folgende Inhalte und Ziele verbunden:

- Nutzung und Ausbau der bereits durch Cuparla geschaffenen Infrastruktur für die Betreuung und Informationsversorgung von Oberbürgermeister, Bürgermeistern und Amtsleitungen

- Schaffung einer gemeinsamen Telekooperationsumgebung für Gemeinderat und Führungskräfte, die ein kooperatives Arbeiten ermöglicht.

- Aufbau eines Ratsinformationssystems, das als Controllinginstrument aller Aufträge aus dem Gemeinderat dient und ein standardisiertes Berichtswesen integriert

- Integration statistischer Auswertungen und Kennzahlen

- Ausbau des vorhandenen Intranets zur Basisinformationsversorgung

- Im Ergebnis soll eine Verwesentlichung und Beschleunigung von Entscheidungen und für den Bürger eine Qualitätsverbesserung erzielt werden.

Die Etablierung von Telekooperationstechnologie als gemeinsame Basis für die Zusammenarbeit von Gemeinderat und Verwaltungsführung kann nur gelingen, wenn die erfolgskritische Masse an Personen auf beiden Seiten erreicht wird (zur kritischen Masse vgl. z.B. 
[Markus 1990]). Auf der Seite des Gemeinderats ist dies bereits erfolgt. Zwangsläufig entsteht so eine Wettbewerbssituation: Nur Führungskräfte, die ihrerseits aktiv die angebotene Technik nutzen, können die Aufträge des Gemeinderats in der geforderten Zeit erfüllen. Gegenüber dem Bürger wie auch dem Gemeinderat wird so die Nutzung von Telekooperation ein wesentlicher Prestige- und Erfolgsfaktor.

\section{$5 \quad$ Einführung von Telekooperation für das mittlere Management}

Die Landeshauptstadt Stuttgart hat Ende 1995 parallel zu Cuparla die Einführung eines Sitzungsdienstverfahrens beschlossen. Ziel war ein einheitliches Verfahren zur Erstellung von Vorlagen, das stadtweit auf allen damals gängigen Betriebsystemplattformen (MS DOS/WFW, IBM OS/2, später MS Windows NT) und Netzwerken (TCP-IP, IPX-SPX) eingesetzt werden konnte. Letztlich wurde ein Verfahren des Rechenzentrum Frankens auf der Basis von Lotus Notes ausgewählt. Die nachfolgende Abbildung zeigt die Eingangshalle der aktuellen Version 2.0:

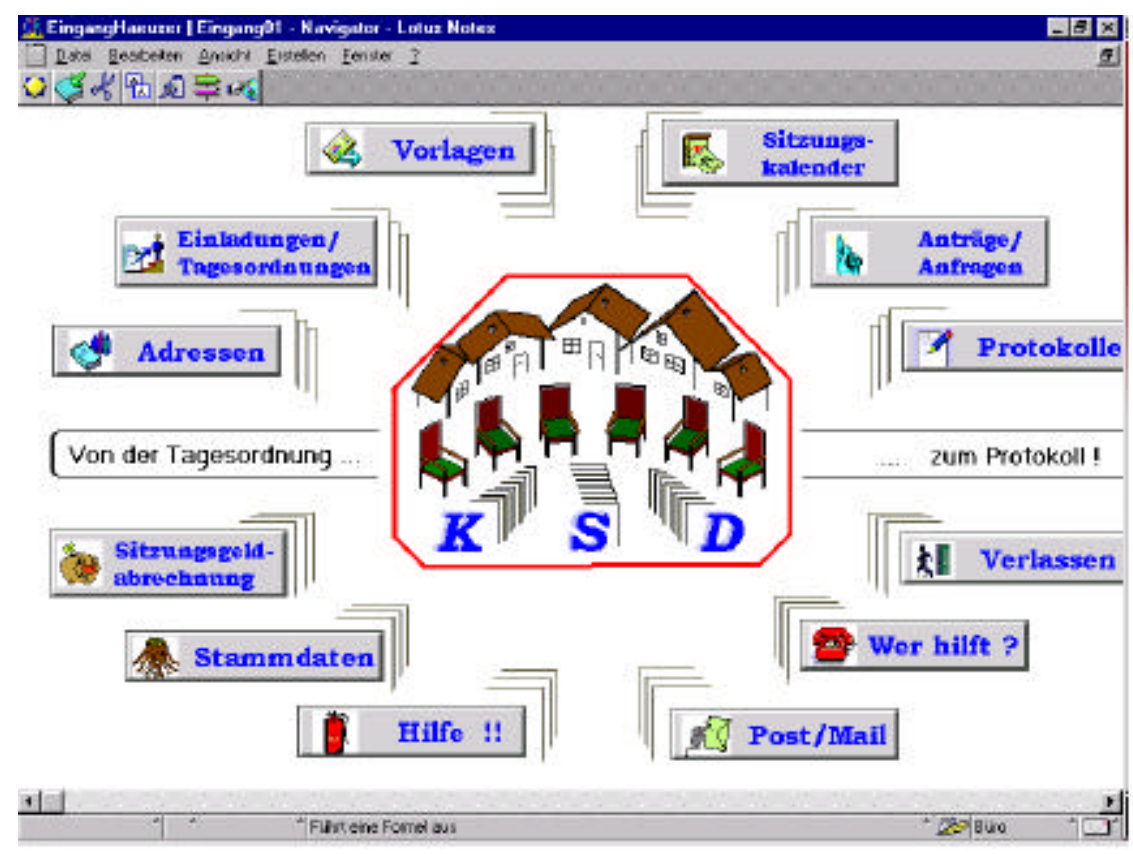

Abbildung 3: Der Kommunale Sitzungsdienst

Sämtliche Anträge, Anfragen (aus Cuparla), Tagesordnungen, Protokolle und Vorlagen werden in diesem Verfahren abgebildet. Zudem sind die Kurzfassungen der Vorgänge der 
letzten 10 Jahre in einer separaten Notes-Datenbank verfügbar. Öffentliche Dokumente werden über einen Domino-Server auch im städtischen Intranet angeboten und sollen zu einem späteren Zeitpunkt für den Ausbau der Internet-Präsenz (Bürgerinformation) der Landeshauptstadt Stuttgart genutzt werden.

Das Verfahren unterstiutzt die gesamte elektronische Erstellung und Bearbeitung von Vorlagen. Dabei kann der jeweilige Ersteller alle mitzeichnenden Referate und Bearbeiter vorgeben. Die Version 2.0 beinhaltet darüber hinaus ein umfangreiches Wiedervorlagemodul, das eine laufende Statuskontrolle über alle Vorgä nge ermöglicht.

Obwohl mittlerweile nahezu jedes Fachamt und Referat über einen Anschluß an den Kommunalen Sitzungsdienst verfügt, werden auch heute noch häufig Vorlagen nicht mit dem Sitzungsdienstverfahren erstellt, sondern im Nachgang zum Beschluß als Word-Datei aufwendig in das System eingepflegt. Die Ursachen hierfür sind vielfältig:

- Aufgrund der nur beschränkt verfügbaren Administrationspersonals können nicht alle vorlagenerstellenden Stellen versorgt werden.

- Für die Erstellung von Vorlagen genügt - funktional betrachtet - ein einfacher Texteditor. Die ausführliche Begründung und spezielle Dokumente wie Pläne oder Berechnungen können an das Vorlagendokument in verschiedenen Formaten (MS Word, Excel etc.) angehängt werden. Hier bestehen teilweise Akzeptanzprobleme, da der gängige WordEditor klare Präferenz genießt.

- Mit dem Kommunalen Sitzungsdienst kann der Status eine Vorlage transparent dargestellt werden, was bislang nur sehr schwer möglich war. Damit werden aber verwaltungsintern über Jahre eingespielte „Abläufe“ verändert. Die per Verfahren vorgegebene Bearbeitungs- und Mitzeichnungsabfolge stößt damit zwangsläufig auf Widerstand, z.T. verbunden mit einer generellen Ablehnung von Technik und wird dann häufig wieder durch die konventionelle „Papiermethode“ ersetzt. Die Mitzeichnung - ein primär sequentieller Vorgang - kann technikunterstützt nur funktionieren, wenn alle daran Beteiligten das Verfahren annehmen und ,leben“.

Die Einführungsstrategie für die nun anstehende Version $2.0 \mathrm{mu}$ diese Erfahrungen berücksichtigen. Letztlich wird aber ein stärkeres Einwirken der Referatsbereiche auf die angeschlossenen Fachämter bzw. des Gemeinderats notwendig werden. Die Vorteile des Verfahrens sind unbestritten. Die damit verbundene Transparenz wird aber zu Widerständen bei der Nutzung führen. Letztlich wird der Grad eines kooperativen Zusammenwirkens von Rat und Verwaltung - im Interesse der Bürger - aber vom gegenseitigen Vertrauen bestimmt.

\section{$6 \quad$ Einführung von Telekooperation für den operativen Verwaltungsbereich}

Es ist zu erwarten, daß der Benutzerservice der Landeshauptstadt Stuttgart nicht in dem Maße personell verstärkt werden kann, wie die Zahl der PC-Nutzer und deren Ansprüche bzgl. Betreuungsqualität wachsen werden. Aus dieser Erwartung heraus wurde die Philoso- 
phie „Mobiler Benutzerservice“ geboren, die Synonym und Leitbild für das Selbstverständnis und die räumlich und zeitlich flexible Einsatzbereitschaft der Mitarbeiter und Mitarbeiterinnen werden soll.

Ein wesentliches Kriterium für die Erhöhung der Produktivität in diesem Bereich ist die durchgängige Abbildung wesentlicher Geschäftsprozesse in einer Softwarelösung - ohne jeglichen Medienbruch. Die aktuelle Situation ist gekennzeichnet durch:

- Die häufige Mehrfacherfassung von Daten (z.B. Bestands- und Auftragsdaten) vor Ort und in der Zentrale.

- Das vorhandene Host-basierende Verfahren für das Bestandsmanagement bietet einen unzureichenden, z.T. lückenhaften oder gar falschen Überblick über die aktuell installierte Hard- und Software eines Amtes. Der Benutzerservice muß diese Informationen während eines Servicefalls dann erneut erheben, was Reaktionszeit und Kosten erhöht.

- Keine zentrale Sammlung von Know-how über die Lösung bestimmter Vorgänge (z.B. Reparatur eines PCs). Ein Qualitätssicherungssystem fehlt.

- $\quad$ Eine Workflowlösung, die eine Statusverfolgung und Delegation innerhalb eines Servicefalles erlauben würde, ist bis heute noch nicht vorhanden.

Das Kundenportfolio ist durch räumliche Verteilung und unterschiedliche Arbeitszeiten gekennzeichnet. Die derzeit noch vergleichsweise starre Personaldisposition, die aus einem mangelnden Überblick über den Aufenthaltsort und die Auslastung einzelner Personen beim Benutzerservice resultiert, genügt dem Anspruch einer im Sinne der Verwaltungsreform zunehmend als Dienstleister proklamierten Serviceeinheit nicht mehr.

Der Benutzerservice wird der Erwartungshaltung nur gerecht werden können, wenn alle Servicemitarbeiter und -mitarbeiterinnen die notwendigen Informationen über Hard- und Software, bisher aufgetretene Probleme, deren Lösung und die komplette Auftragsabwicklung möglichst in einer Arbeitsumgebung auf ihrem PC bzw. vor Ort auf einem Notebook abrufen können. Die aus dem Projekt Cuparla bekannte Oberfläche würde sich bei entsprechender Modifikation sehr gut dafür eignen. Das „Raumdesign“ könnte dabei vor allem für die Statusverfolgung eines Serviceauftrags ideal eingesetzt werden.

Folgende Datenbanken müßten u.a. implementiert werden:

Bestandsdatenbank: Informationen über Hard- und Software werden mit dem Notebook vor Ort aktualisiert und mit dem zentralen Server synchronisiert. Die zentrale Disposition für einen Serviceeinsatz verfügt so über alle erforderlichen Informationen. Hardwareinformationen (z.B. Typenbezeichnung und Seriennummer) könnten z.T. direkt von den Lieferanten in elektronischer Form bezogen werden. Teilweise sind diese Informationen bereits vor der Lieferung direkt über das Internet abrufbar.

Know-how-Datenbank: Probleme und Lösungen müssen strukturiert gesammelt werden. Damit soll vermieden werden, daß zeitlich versetzt mehrfach an der gleichen Problemstellung gearbeitet wird. Die gesammelten Erfahrungen - z.B. über bestimmte Installationsabläufe - können zu Handlungsanweisungen verdichtet werden, die die Basis für ein Qualitätssicherungssystem bilden. 
Treiberdatenbank: Die für den Serviceeinsatz erforderlichen Treiber sollen im jeweils aktuellen Releasestand zentral gepflegt werden.

Prozeßdatenbank: Die Anwender werden bei einem technischen Problem oder einer Frage zunächst bei der zentralen Hotline anrufen. Der Anruf wird hier erfaßt und - falls keine sofortige Lösung möglich ist - vom Disponenten an den Second-Level-Support als „Ticket“ weitergeleitet (Einsatz des „Raumdesigns“ [vgl. Schwabe et al. 1997]). Der jeweilige Status und die zuständige Person können abgerufen werden. Alle Servicefälle werden in ihrem gesamten Ablauf erfaßt. Zudem werden Auswertungen über die Häufigkeit bestimmter Störungen und Empfehlungen für künftige Beschaffungen ermöglicht. Die Transparenz, die gerade mit dieser Datenbank geschaffen wird, könnte das Vertrauen in die Leistungsfähigkeit des Benutzerservices stärken. Auf der anderen Seite können Kennzahlen z.B. für die durchschnittliche Bearbeitungszeit von bestimmten Störfällen ermittelt werden. Die Transparenz erzeugt damit zwangsläufig auch eine bessere Vergleichbarkeit mit internen wie externen Anbietern. Der „totale Wettbewerb“ wie ihn Städte wie z.B. Christchurch in Neuseeland bereits vorleben, wird aber auch in deutschen Kommunen zunehmend in die Überlegungen zur Verwaltungsreform einbezogen.

Für die Implementierung von „Mobiler Benutzerservice“ ist ein Leistungsanreizsystem zu etablieren, das trotz der bescheidenen finanziellen Möglichkeiten des öffentlichen Dienstes, Leistung zu honorieren, den bei allen Beteiligten vorhandenen Wunsch nach Verbesserung der Situation aufgreift und als treibende Kraft für die notwendigen organisatorischen Änderungen nutzt. Ansonsten ist der Wettbewerbsnachteil gegenüber externen Anbietern nicht auszugleichen. Wesentliche Elemente hierfür sind:

- Die bessere Vereinbarkeit von Beruf und Familie. Flexiblere Arbeitszeiten im Sinne der Kunden müssen auf der anderen Seite für eine auf die persönliche Lebenssituation der Mitarbeiter abgestimmte Freizeitgestaltung genutzt werden können.

- Die Nutzung von Telekooperationstechnologie muß als exklusives, zusätzliches „Bonbon“ empfunden werden. Die Mobilität, die mit dem Einsatz von Notebooks, Handys, PDAs etc. abverlangt wird, ist für die Servicemitarbeiter zumeist mit einer erhöhten Einsatzfrequenz verbunden. Für viele Servicemitarbeiter ist aber gerade der Besitz solchen Equipments von großer, persönlicher Bedeutung.

- Die Stellensituation führt zwangsläufig zu einem stärkeren Wettbewerb um attraktive Positionen. Die Beherrschung von Telekooperation in allen technischen wie auch sozialen Facetten wird zunehmend zum Wettbewerbsfaktor.

\section{Zusammenfassung}

Telekooperation bedeutet nicht nur eine technische, sondern auch eine organisatorische Herausforderung. Da die Telekooperation nur dann erfolgreich ist, wenn alle wesentlichen an der Kooperation beteiligten Personen es nutzen, kommt der Motivation der Akteure bei der Einführung eine Schlüsselrolle. Dabei spielen mikropolitische Faktoren eine mindestens so bedeutende Rolle wie rationalökonomische Faktoren. Wir konnten zeigen, daß - richtig 
eingesetzt - Wettbewerb auf allen Ebenen eine fördernde Rolle für die Einführung von Telekooperation spielen kann. In der öffentlichen Verwaltung ist Wettbewerb dann ein fruchtbarer Anreiz, wenn er an der Mikropolitik ansetzt.

\section{Literatur}

Krcmar, H.: Informationsmanagement, Springer; Heidelberg u.a. 1997.

Landeshauptstadt Stuttgart: Gemeinderatsdrucksache GRDrs 264/1998 zur Verwaltungsreform, Stuttgart 1998.

Markus, M.: Toward a 'critical mass' theory of interactive media. In: Fulk, J.; Steinfeld, C.: Organizations and communication technology, Sage, Beverly Hills 1990, S. 194-218.

Murawski, K.: Die Verantwortung des Rates im Reformprozeß. Vortrag auf der Fachkonferenz 'Bürger -Politik-Verwaltungsreform' am 12/13.3. 1998 in Koblenz.

Ortmann, G.; Windeler, A.; Becker, A.; Schulz, H.: Computer und Macht in Organisationen. Westdeutscher Verlag, Opladen 1990.

Schwabe, G; Krcmar, H: Wettbewerb als Einführungsstrategie von Telekooperation für Entscheidungsträger - Erfahrungen aus dem Projekt Cuparla. In: Wirtschaftsinformatik, Vol. 40, Nr. 3. 1998, S. 200-204.

Schwabe, G., Hertweck, D.; Krcmar, H.: Partizipation und Kontext bei der Erstellung einer Telekooperationsumgebung. In: Jarke, M.; Pasedach, K.; Pohl, K.: Informatik 97 - Informatik als Innovationsmotor, Springer, Heidelberg. et al. 1997, S. 370-379.

Schwabe, G., Vöhringer, B.: Computerunterstützung der Parlamentsarbeit - ein Baustein zum Umbau der Verwaltung. In: Verwaltung und Management Vol. 4, Nr. 3, 1998, S. 140-147.

Schwabe, G.: Telekooperation für den Gemeinderat. Eingereichte Habilitationsschrift an der Universität Hohenheim, Stuttgart 1998.

Schwabe G.: Informationsmanagement für den Gemeinderat: Erscheint in: Hummeltenberg, W.: Information Management for Business Intelligence, Proceedings der Frühjahrstagung der Wissenschaftlichen Kommission Wirtschaftsinformatik in Hamburg, Vieweg 1998b.

Schwarzer, B.; Zerbe, S.; Krcmar, H.: An Eclectic Framework For Understanding New Organisational Forms. In: social sciences, COST A3, Volume 3, Management and network technology. Hrsg.: European Commission, Office for Official Publications of the European Communities, Luxembourg, 1997, S. 85-97. 